\title{
Diameter and diametrical pairs of points in ultrametric spaces
}

\author{
D. Dordovskyi, O. Dovgoshey and E. Petrov
}

\begin{abstract}
Let $\mathcal{F}(X)$ be the set of finite nonempty subsets of a set $X$. We have found the necessary and sufficient conditions under which for a given function $\tau: \mathcal{F}(X) \rightarrow \mathbb{R}$ there is an ultrametric on $X$ such that $\tau(A)=\operatorname{diam} A$ for every $A \in \mathcal{F}(X)$. For finite nondegenerate ultrametric spaces $(X, d)$ it is shown that $X$ together with the subset of diametrical pairs of points of $X$ forms a complete $k$-partite graph, $k \geqslant 2$, and, conversely, every finite complete $k$-partite graph with $k \geqslant 2$ can be obtained by this way. We use this result to characterize the finite ultrametric spaces $(X, d)$ having the minimal $\operatorname{card}\{(x, y): d(x, y)=\operatorname{diam} X, x, y \in X\}$ for given card $X$.
\end{abstract}

\section{Mathematics Subject Classification (2000): 54E35.}

Key words: ultrametric space, complete $k$-partite graph, diameter of sets in ultrametric spaces.

\section{Introduction}

A metric space $(X, d)$ is ultrametric if the following ultrametric inequality

$$
d(x, y)=d(x, z) \vee d(z, y)
$$

holds for all $x, y, z \in X$, where we put

$$
d(x, z) \vee d(z, y)=\max \{d(x, z), d(z, y)\} .
$$

The ultrametric spaces and, in particular, the finite ultrametric spaces play an important role for the modern physics, data analysis, mathematical taxonomy and many others branches of the knowledge (see, for example, [1]). In particular, the following fact seems to be fundamental in the theory of hierarchical models:

Theorem 1.1 (see [2]). For every dendrogram $\mathcal{D}$ (= nested family of partitions) over a finite set $X$ there is an ultrametric $d$ on $X$ representing this $\mathcal{D}$. 
We do not define more exactly the sense of the word "representing" in Theorem 1.1 but note that the "dual form" of this theorem will be given in Theorem 3.11 below.

There exist also the significant relations between (finite) ultrametric spaces and many mathematical constructions. The well known example is the canonical representation of finite ultrametric spaces by the weighted rooted trees. Note that this representation can be generalized for the case of representation of quasi-ultrametric finite spaces by the weighted digraphs, [3]. It is also shown in [3] that finite ultrametric spaces can be viewed as $k$-person positional games. An isomorphism of categories of ultrametric spaces and complete, atomic, tree-like, real graduated lattices was proved in [4].

Some basic results in the theory of (finite) ultrametric spaces were obtained quite recently. The isometry groups of finite ultrametric spaces are completely characterized in [5]. A set of necessary and sufficient conditions under which the Cartesian products of metric spaces are ultrametric was found in [6]. The counterpart of Gromov-Hausdorff metric in the ultrametric spaces was studied in [7], [8]. The infinitesimal structure of pointed metric spaces having ultrametric "tangent" spaces was described in [9].

In the present paper we solve the following two problems.

Let $(X, \rho)$ be a metric space and let $A$ be a subset of $X$. Recall that the diameter of $A$ is the quantity

$$
\operatorname{diam} A=\sup \{\rho(x, y): x, y \in A\} .
$$

We shall say that a two-element subset $\{a, b\}$ of $A$ is a $\rho$-diametrical pair for $A$ if $\rho(a, b)=\operatorname{diam} A$. Write $\mathbb{R}:=(-\infty, \infty), \mathbb{R}^{+}:=[0, \infty)$ and $\mathcal{F}(Y)$ for the set of all nonempty finite subsets of a set $Y$.

(a) Under what conditions on a function $\tau: \mathcal{F}(Y) \rightarrow \mathbb{R}$ there is an ultrametric $\rho: Y \times Y \rightarrow \mathbb{R}^{+}$such that

$$
\operatorname{diam} A=\tau(A)
$$

for every $A \in \mathcal{F}(Y)$ ?

(b) Characterize the structure of simple graphs $G=(V, E)$ for which there is an ultrametric $\rho$ defined on the vertex set $V$ such that the edge set $E$ coincides with the set of $\rho$-diametrical pairs.

\section{Diameter in ultrametric spaces}

The following theorem describes the characteristic properties of diameters of finite subsets in ultrametric spaces. 
Theorem 2.1. Let $X$ be a nonempty set. The following two statements are equivalent for every function $\tau: \mathcal{F}(X) \rightarrow \mathbb{R}$.

(i) For all $A, B, C \in \mathcal{F}(X)$ the function $\tau$ satisfies the conditions:

$\left(i_{1}\right)(\tau(A)=0) \Leftrightarrow(\operatorname{card} A=1) ;$

$\left(i_{2}\right)$

$$
\tau(A \cup B) \leqslant \tau(A \cup C) \vee \tau(C \cup B) .
$$

(ii) There is an ultrametric $\rho: X \times X \rightarrow \mathbb{R}^{+}$such that

$$
\tau(A)=\operatorname{diam} A
$$

for every $A \in \mathcal{F}(X)$.

Remark 2.2. Conditions $\left(i_{1}\right)$ and $\left(i_{2}\right)$ are closely related to the similar conditions from the paper of P. Balk [10]. In fact, Balk's paper was a starting point in our consideration.

Remark 2.3. Let (ii) hold. Properties $\left(\mathrm{i}_{1}\right)$ and $\left(\mathrm{i}_{2}\right)$ imply that the map $\tau: \mathcal{F}(X) \rightarrow \mathbb{R}$ is isotonic and nonnegative. Indeed, substituting of $A$ for $B$ into (2.1) we see that

$$
\tau(A) \leqslant \tau(C)
$$

if $A \subseteq C$. Putting $A=\{c\}$ in the last inequality, where $c$ is an arbitrary element of $C$, and using $\left(\mathrm{i}_{1}\right)$ we obtain $\tau(C) \geqslant 0$ for $C \in \mathcal{F}(X)$.

Proof of Theorem [2.1. (ii) $\Rightarrow($ i). Let $\rho$ be an ultrametric on $X$ such that

$$
\tau(A)=\operatorname{diam} A, \quad A \in \mathcal{F}(X) .
$$

Property $\left(\mathrm{i}_{1}\right)$ is evident. To prove $\left(\mathrm{i}_{2}\right)$ suppose that

$$
\max _{x, y \in A \cup B} \rho(x, y)=\max _{x, y \in A} \rho(x, y) .
$$

It implies

$$
\begin{gathered}
\operatorname{diam}(A \cup B)=\max _{x, y \in A} \rho(x, y) \leqslant \max _{x, z \in A \cup C} \rho(x, z) \\
\leqslant \max _{x, z \in A \cup C} \rho(x, z) \vee \max _{y, z \in B \cup C} \rho(y, z)=\operatorname{diam}(A \cup C) \vee \operatorname{diam}(C \cup B) .
\end{gathered}
$$

Similarly we obtain (2.1) if $\max _{x, y \in A \cup B} \rho(x, z)=\max _{x, y \in B} \rho(x, y)$.

Suppose now that $\max _{x, y \in A \cup B} \rho(x, y)=\max _{x \in A, y \in B} \rho(x, y)=\rho(a, b)$ where $a \in$ $A, b \in B$. Then for every $c \in C$ we have

$$
\operatorname{diam}(A \cup B)=\max _{x \in A, y \in B} \rho(x, y)=\rho(a, b) \leqslant \rho(a, c) \vee \rho(b, c)
$$




$$
\leqslant \max _{x \in A, z \in C} \rho(x, z) \vee \max _{y \in B, z \in C} \rho(y, z) \leqslant \operatorname{diam}(A \cup C) \vee \operatorname{diam}(C \cup B) .
$$

Property $\left(\mathrm{i}_{2}\right)$ follows.

$(\mathrm{i}) \Rightarrow(\mathrm{ii})$. Let $\tau: \mathcal{F}(X) \rightarrow \mathbb{R}$ be a function satisfying $\left(\mathrm{i}_{1}\right)$ and $\left(\mathrm{i}_{2}\right)$. Let us define a function $\rho$ on $X \times X$ by the rule

$$
\rho(x, y):=\tau(\{x, y\}), \quad x, y \in X
$$

where $\{x, y\}$ is the set with the elements $x$ and $y$. Property $\left(\mathrm{i}_{1}\right)$ implies that $\rho(x, y)=0$ if and only if $x=y$. Moreover, $\rho$ is nonnegative (see Remark 2.3). The ultrametric inequality $\rho(a, b) \leqslant \rho(a, c) \vee \rho(c, b)$ follows from (2.1) with $A=\{a\}, B=\{b\}$ and $C=\{c\}$. Thus, $\rho$ is an ultrametric. It still remains to prove (2.2) for $A \in \mathcal{F}(X)$ with card $A \geq 3$. Let $A=\left\{x_{1}, \ldots, x_{n}\right\}$. Then for $j=1, \ldots, n$ we obtain

$$
\begin{gathered}
\tau(A)=\tau\left(\left\{x_{1}, \ldots, x_{n-1}\right\} \cup\left\{x_{n}\right\}\right) \leqslant \tau\left(\left\{x_{1}, \ldots, x_{n-1}\right\} \cup\left\{x_{j}\right\}\right) \vee \tau\left(\left\{x_{n}, x_{j}\right\}\right) \\
=\tau\left(\left\{x_{1}, \ldots, x_{n-1}\right\}\right) \vee \rho\left(x_{n}, x_{j}\right) \leqslant \tau\left(\left\{x_{1}, \ldots, x_{n-1}\right\}\right) \vee\left(\max _{1 \leqslant j \leqslant n} \rho\left(x_{n}, x_{j}\right)\right) .
\end{gathered}
$$

The repetition of this procedure gives

$$
\tau(A) \leqslant \bigvee_{1 \leqslant i \leqslant j \leqslant n} \rho\left(x_{i}, x_{j}\right)=\operatorname{diam} A
$$

As was shown in Remark $2.3 \tau$ is an isotonic map. Consequently, if $x_{i}, x_{j}$ is a diametrical pair of points of $A$, then

$$
\tau(A) \geqslant \tau\left(\left\{x_{i}, x_{j}\right\}\right)=\rho\left(x_{i}, x_{j}\right)=\operatorname{diam} A .
$$

Thus, (2.1) holds for every $A \in \mathcal{F}(X)$.

Remark 2.4. The second part of Theorem 2.1 shows, in particular, that for every function $\tau: \mathcal{F}(X) \rightarrow \mathbb{R}$ satisfying $\left(\mathrm{i}_{1}\right)-\left(\mathrm{ii}_{2}\right)$ there is a unique ultrametric $\rho: X \times X \rightarrow \mathbb{R}^{+}$such that $\rho(x, y)=\tau(\{x, y\})$ for all $x, y \in X$.

Theorem 2.1 can be generalized to the case of functions $\tau$ defined also on infinite subsets of $X$.

Let $\mathfrak{Y}(X)$ be the set of nonempty subsets of $X$.

Theorem 2.5. The following two statements are equivalent for every function $\tau: \mathfrak{Y}(X) \rightarrow \mathbb{R}$.

(i) For all $A, B, C \in \mathfrak{Y}(X)$ the function $\tau$ satisfies conditions ( $\left.i_{1}\right)$ and $\left(i_{2}\right)$ from Theorem 2.1 and the following condition 
$\left(i_{3}\right)$

$$
\tau(A) \leqslant \sup \{\tau(B): B \in \mathcal{F}(A)\} .
$$

(ii) There is an ultrametric $\rho: X \times X \rightarrow \mathbb{R}^{+}$such that $\tau(A)=\operatorname{diam} A$ for every $A \in \mathfrak{Y}(X)$.

This theorem can be obtained as a consequence of Theorem 2.1. Indeed, since $\mathcal{F}(X) \subseteq \mathfrak{Y}(X)$, condition (i) of Theorem 2.5 implies condition (i) of Theorem 2.1. Consequently, the restriction $\left.\tau\right|_{\mathcal{F}(X)}$ is a diameter of finite sets for some ultrametric $\rho: X \times X \rightarrow \mathbb{R}^{+}$. If card $A=\infty$, then the equality $\tau(A)=\operatorname{diam} A$ follows from the same equality with card $A<\infty$ by application of condition $\left(i_{3}\right)$. The details of the proof we leave to the reader.

Example 2.6. Let $X$ be an infinite set. Define a function $\tau: \mathfrak{Y}(X) \rightarrow \mathbb{R}$ as

$$
\tau(A)= \begin{cases}0 & \text { if } \operatorname{card} A=1, \\ 1 & \text { if } 1<\operatorname{card} A<\infty \\ 2 & \text { if } \operatorname{card} A=\infty .\end{cases}
$$

It is easy to prove that condition $\left(i_{3}\right)$ does not hold but $\left(i_{1}\right)$ and $\left(i_{2}\right)$ take place for this $\tau$. Thus, $\left(\mathrm{i}_{1}\right)$ and $\left(\mathrm{i}_{2}\right)$ do not imply $\left(\mathrm{i}_{3}\right)$.

The key property of ultrametric spaces is that their balls are either disjoint or comparable w.r.t. the set-theoretic inclusion. Let us extend this property to the "balls" in $\mathcal{F}(X)$. Let $\tau$ be a real-valued function on $\mathcal{F}(X)$ satisfying $\left(\mathrm{i}_{1}\right)$ and $\left(\mathrm{i}_{2}\right)$. For $r>0$ and $A \in \mathcal{F}(X)$ define a "closed" ball

$$
B_{r}(A):=\{C \in \mathcal{F}(X): \tau(A \cup C) \leqslant r\}
$$

with a center $A$ and radius $r$.

The following properties are evident:

- $B_{r}(A)=\varnothing$ if and only if $r<\tau(A)$;

- Every nonempty subset $C$ of the set $A$ belongs to the ball $B_{r}(A)$ if this ball is nonempty;

- $B_{r_{1}}(A) \supseteq B_{r_{2}}(A)$ if $r_{1} \geqslant r_{2}$.

Proposition 2.7. Let $X$ be a nonempty set and $\tau: \mathcal{F}(X) \rightarrow \mathbb{R}$ be a function satisfying condition (i) of Theorem [2.1. Then for every ball $B_{r}(A)$ and every $C \in \mathcal{F}(X)$ we have

$$
B_{r}(C)=B_{r}(A)
$$


if $C \in B_{r}(A)$, furthermore, for each pair $B_{r_{1}}\left(A_{1}\right), B_{r_{2}}\left(A_{2}\right)$ with $r_{1} \geqslant r_{2}$ we have either

$$
B_{r_{1}}\left(A_{1}\right) \cap B_{r_{2}}\left(A_{2}\right)=\varnothing \text { or } B_{r_{2}}\left(A_{2}\right) \subseteq B_{r_{1}}\left(A_{1}\right) .
$$

Proof. Let $C$ belong to $B_{r}(A)$. To prove the inclusion

$$
B_{r}(C) \subseteq B_{r}(A)
$$

suppose that $Y \in B_{r}(C)$, i.e. $\tau(Y \cup C) \leqslant r$. Since $C \in B_{r}(A)$, we have $\tau(A \cup C) \leqslant r$. Using $\left(\mathrm{i}_{2}\right)$ we obtain $\tau(A \cup Y) \leqslant \tau(A \cup C) \vee \tau(C \cup Y)$. Consequently, $\tau(A \cup Y) \leqslant r$ holds, so (2.7) follows. To prove (2.5) it remains to show that

$$
B_{r}(A) \subseteq B_{r}(C)
$$

For every $Z \in B_{r}(A)$ we have the inequality

$$
\tau(A \cup Z) \leqslant r .
$$

Since $C \in B_{r}(A)$, we also obtain

$$
\tau(A \cup C) \leqslant r .
$$

Inequalities (2.9), (2.10) and condition $\left(i_{1}\right)$ imply

$$
\tau(C \cup Z) \leqslant \tau(C \cup A) \vee \tau(A \cup Z) \leqslant r \vee r=r .
$$

Thus $Z \in B_{r}(C)$, that implies (2.8).

Suppose now that $B_{r_{1}}\left(A_{1}\right)$ and $B_{r_{2}}\left(A_{2}\right)$ be some balls such that $r_{1} \geqslant r_{2}$. If $B_{r_{1}}\left(A_{1}\right) \cap B_{r_{2}}\left(A_{2}\right) \neq \varnothing$, then there is $C \in B_{r_{1}}\left(A_{1}\right) \cap B_{r_{2}}\left(A_{2}\right)$. As we already know, the equalities $B_{r_{1}}\left(A_{1}\right)=B_{r_{1}}(C)$ and $B_{r_{2}}\left(A_{2}\right)=B_{r_{2}}(C)$ hold. Consequently, the inequality $r_{1} \geqslant r_{2}$ implies (2.6).

Using Proposition 2.7 and the characteristic property of bases for topological spaces [11, p. 21] we see that the family

$$
\mathbf{B}(X):=\left\{B_{r}(A): A \in \mathcal{F}(X), r \geqslant \tau(A)\right\}
$$

is a base for some topology on $\mathcal{F}(X)$. It is well known that the family of closed balls in each ultrametric space forms a clopen base for the space, but, in general, it is not the case for the base $\mathbf{B}(X)$.

Indeed, suppose that $a, b, c, d$ are some points of $X$ such that

$$
\tau(\{a, b\})<\tau(\{c, d\})=\sup \{\tau(A): A \in \mathcal{F}(X)\}
$$


and the ball

$$
B_{r_{1}}(\{a, b\}), \quad r_{1}:=\tau(\{a, b\})
$$

is closed in the topology generated by the base $\mathbf{B}(X)$. The inequality in (2.11) and (2.4) imply

$$
\{c, d\} \in \mathcal{F}(X) \backslash B_{r_{1}}(\{a, b\}) .
$$

By the supposition the set $\mathcal{F}(X) \backslash B_{r_{1}}(\{a, b\})$ is open. Since $\mathbf{B}(X)$ is a base, there is $B_{r_{2}}(A) \in \mathbf{B}(X)$ such that

$$
\{c, d\} \in B_{r_{2}}(A) \subseteq \mathcal{F}(X) \backslash B_{r_{1}}(\{a, b\}) .
$$

By Proposition 2.7, we have the equality $B_{r_{2}}(A)=B_{r_{2}}(\{c, d\})$. The smallest $r \in \mathbb{R}^{+}$for which $\{c, d\} \in B_{r}(\{c, d\})$ equals $\tau(\{c, d\})$. Consequently, the inequality $r_{2} \geqslant \tau(\{c, d\})$ holds. The last inequality and the inclusion in (2.12) show that

$$
\{a, b\} \notin B_{r_{3}}(\{c, d\})
$$

where $r_{3}:=\tau(\{c, d\})$. The equality in (2.11) and (2.4) imply $A \in B_{r_{3}}(\{c, d\})$ for every $A \in \mathcal{F}(X)$, contrary to (2.13).

\section{Diametrical pairs of points in ultrametric spaces}

To formulate the main results of the present section we recall the basic definitions from the graph theory (see [12] for all terms that will be used without explicit formulations).

A graph $G$ is an ordered pair $(V, E)$ consisting of a set $V=V(G)$ of vertices and a set $E=E(G)$ of edges. In this paper we consider only simple graphs, so each edge is an unordered pair of distinct vertices and $E(G)$ is a set of two-element subsets of $V(G)$.

Definition 3.1. Let $G$ be a simple graph and let $k$ be a cardinal number. The graph $G$ is $k$-partite if the vertex set $V(G)$ can be partitioned into $k$ nonvoid disjoint subsets, or parts, in such a way that no edge has both ends in the same part. A k-partite graph is complete if any two vertices in different parts are adjacent.

We shall say that $G$ is a complete multipartite graph if there is $k \geqslant 1$ such that $G$ is complete $k$-partite, cf. [13, p. 14].

Remark 3.2. It is easy to see that 1-partite graph $G$ is empty in the sense that no two vertices are adjacent. 
To simplify the formulations we consider the cases of finite and infinite ultrametric spaces separately.

Theorem 3.3. Let $(X, \rho)$ be an ultrametric space with card $X=\infty$ and let $G$ be a graph such that $V(G)=X$ and

$$
(\{u, v\} \in E(G)) \Leftrightarrow(\{u, v\} \text { is a } \rho \text {-diametrical pair of points for } X) \text {. }
$$

Then $G$ is complete multipartite graph. Conversely, if $G=(V, E)$ is a complete multipartite graph with card $V=\infty$, then there is an ultrametric $\rho: V \times V \rightarrow \mathbb{R}^{+}$such that $E$ is the set of $\rho$-diametrical pairs of points for $V$.

Theorem 3.4. Let $(X, \rho)$ be an ultrametric space with $2 \leqslant \operatorname{card} X<\infty$ and let $G$ be a graph such that $V(G)=X$ and condition (3.1) holds. Then $G$ is a complete $k$-partite graph with $k \geqslant 2$. Conversely, if $G=(V, E)$ is a finite (card $V<\infty)$ complete multipartite graph with $k \geqslant 2$, then there is an ultrametric $\rho: V \times V \rightarrow \mathbb{R}^{+}$such that $E$ is the set of $\rho$-diametrical pairs of points for $V$.

The proof of these theorems based on the following three lemmas.

Lemma 3.5. Let $(X, \rho)$ be an ultrametric space with card $X \geqslant 2$. Let us define the binary relation $\equiv$ on $X$ by the rule

$$
(x \equiv y) \Leftrightarrow(\rho(x, y)<\operatorname{diam} X) .
$$

Then $\equiv$ is an equivalence relation on $X$.

Proof. It is evident that $\equiv$ is symmetric and reflexive. Suppose now that we have $x \equiv y$ and $y \equiv z$ for $x, y, z \in X$. Then, by the ultrametric inequality, we obtain.

$$
\rho(x, z) \leqslant \rho(x, y) \vee \rho(y, z)<(\operatorname{diam} X) \vee(\operatorname{diam} X)=\operatorname{diam} X .
$$

Hence, $\equiv$ is transitive. Thus, $\equiv$ is an equivalence relation.

Let us recall that the complement $\bar{G}$ of a graph $G=(V, E)$ is a graph whose vertex set is $V$ and whose edges are the pairs of distinct nonadjacent vertices of $G$.

Lemma 3.6. Let $G=(V, E)$ be a graph. The following properties are equivalent:

(i) $G$ is a complete multipartite graph; 
(ii) Every connected component of the complement $\bar{G}$ is a complete graph.

Proof. Suppose $G$ is a complete multipartite graph. Let $\left\{Y_{i}: i \in \mathcal{I}\right\}$ be a partition of the vertex set $V$ with an index set $\mathcal{I}$ such that the properties described in Definition 3.1 hold. Let $u, v \in V, u \neq v$. Then $u$ and $v$ are adjacent in $\bar{G}$ if they belong to the same part $Y_{i}$ and nonadjacent in the opposite case. Hence, for every $i \in \mathcal{I}$ the subgraph of $\bar{G}$ induced by $Y_{i}$ is a connected component of $\bar{G}$ and this component is a complete graph. Implication (i) $\Rightarrow$ (ii) follows. The proof of implication (ii) $\Rightarrow(\mathrm{i}$ ) is also straightforward and we omit this here.

Lemma 3.7. Let $X$ be a nonempty set and let $\equiv$ be an equivalence relation on $X$. If card $X=\infty$, then there is an ultrametric $\rho: X \times X \rightarrow \mathbb{R}^{+}$such that (3.2) holds. For finite $X$ with card $X \geqslant 2$, an ultrametric $\rho: X \times X \rightarrow$ $\mathbb{R}^{+}$satisfying (3.2) exists if and only if the relation $\equiv$ is different from the universal relation $\{(x, y): x, y \in X\}$.

Proof. Consider first the case where card $X=\infty$. If $\equiv$ is the universal relation on $X$, then every ultrametric $\rho: X \times X \rightarrow \mathbb{R}^{+}$satisfying the inequality

$$
\rho(x, y)<\operatorname{diam} X
$$

for all $x, y \in X$ satisfies also (3.2). Suppose that $\equiv$ is different from the universal relation, then the partition of the set $X$ on the equivalence classes

$$
\{y: y \equiv x\}, \quad x \in X
$$

contains more than one class. Let us define a function $\rho$ on $X \times X$ as

$$
\rho(x, y):= \begin{cases}0 & \text { if } x=y \\ \frac{1}{2} & \text { if } x \neq y \text { and } x \equiv y \\ 1 & \text { if } x \neq \equiv y\end{cases}
$$

We claim that $\rho$ is an ultrametric on $X$ and (3.2) holds for this $\rho$. To prove that $\rho$ is an ultrametric it is suffice to show that

$$
\rho(x, z) \leqslant \rho(x, y) \vee \rho(y, z)
$$

for pairwise distinct $x, y, z \in X$. The proof is very simple. Since $x, y$ and $z$ are pairwise distinct, inequality (3.5) does not hold if and only if $\rho(x, z)=1$ and $\rho(x, y)=\rho(y, z)=\frac{1}{2}$. Hence, by (3.4), we obtain

$$
x \not \equiv z, \quad x \equiv y \text { and } y \equiv z,
$$


contrary to the transitivity of $\equiv$. Suppose now that card $X<\infty$. If there is an ultrametric $\rho: X \times X \rightarrow \mathbb{R}^{+}$such that (3.2) holds, then using the finiteness of $X$ we can find $x, y \in X$ such that

$$
\rho(x, y)=\operatorname{diam} X \text {. }
$$

Consequently, $\equiv$ is different from the universal relation. The remaining part of the proof can be obtained by a repetition of the arguments given above.

Remark 3.8. In the proof of Lemma 3.7 we use the existence of an ultrametric $\rho$ satisfying (3.3) for all $x, y \in X$. The "explicit" construction of such type metric for arbitrary card $X$ we shall give in Example 3.10 .

Proof of Theorem 3.3. Let $(X, \rho)$ be an ultrametric space, card $X=\infty$ and let $G$ be a graph with $V(G)=X$ and $E(G)$ defined by (3.1). By Lemma 3.6, $G$ is complete multipartite if every connected component of $\bar{G}$ is complete. If $x$ and $y$ are distinct points, then

$$
(\{x, y\} \in E(\bar{G})) \Leftrightarrow(\{x, y\} \notin E(G)) \Leftrightarrow(\rho(x, y)<\operatorname{diam} X) \Leftrightarrow(x \equiv y),
$$

(see (3.2) $)$. By Lemma 3.5, the relation $\equiv$ is an equivalence on $X$. The equivalence classes induced by $\equiv$ form the set of the connected components of $\bar{G}$. All these components are complete because $\equiv$ is transitive.

Conversely, if $G=(V, E)$ is a complete multipartite graph with card $V=$ $\infty$, then, by Lemma [3.6, every connected component of $\bar{G}$ is a complete graph. Let us define the relation $\equiv$ on $V$ as

$$
(x \equiv y) \Leftrightarrow(x \text { and } y \text { belong to the same connected component of } \bar{G}) \text {. }
$$

Since every component is complete, the relation $\equiv$ is an equivalence relation on $V$. By Lemma 3.7, there is an ultrametric $\rho: V \times V \rightarrow \mathbb{R}^{+}$such that

$$
(x \equiv y) \Leftrightarrow(\rho(x, y)<\operatorname{diam} V)
$$

for $x, y \in V$. Consequently, we obtain

$$
(x \not \equiv y) \Leftrightarrow(\{x, y\} \in E(\overline{\bar{G}})) \Leftrightarrow(\{x, y\} \in E(G)) \Leftrightarrow(\rho(x, y)=\operatorname{diam} V) .
$$

Thus, $E(G)$ is the set of $\rho$-diametrical pairs of $(V, \rho)$.

Theorem 3.4 can be proved in much the same way as Theorem 3.3 .

Remark 3.9. The second part of Lemma 3.7 is, in fact, a special particular case of Theorem 1.1. Lemma 3.6 provides a transition of results from complete multipartite graphs to equivalence relations and back. 
Let $(X, d)$ be a nonempty metric space. Following paper [14] we shall say that the set

$$
\operatorname{Spec}(X, d)=\{d(x, y): x, y \in X\}
$$

is the spectrum of $(X, d)$.

Let $A$ be a subset of $\mathbb{R}^{+}$. If $0 \in A$, then there is an ultrametric space $(X, \rho) \operatorname{such}$ that $\operatorname{Spec}(X, \rho)=A$. This result was proved in [14] by the following elegant method. Define $d: A \times A \rightarrow \mathbb{R}^{+}$, setting

$$
d(x, y)=\left\{\begin{array}{c}
x \vee y \text { if } x \neq y \\
0 \text { otherwise }
\end{array}\right.
$$

Then $(A, d)$ is an ultrametric space and $\operatorname{Spec}(A, d)=A$.

Using ultrametric (3.6) we can simply fill the gap in the proof of Lemma 3.7 mentioned in Remark 3.8 .

Example 3.10. Let $X$ be an infinite set, $A_{c}$ a countable infinite subset of $X, A$ a countable infinite subset of $\mathbb{R}^{+}, 0 \in A$,

$$
\sup A \notin A
$$

and let $f: A_{c} \rightarrow A$ be a bijection. For an arbitrary $\varepsilon>0$ define a function $\rho: X \times X \rightarrow \mathbb{R}^{+}$as

$$
\rho(x, y):=\left\{\begin{array}{l}
0 \quad \text { if } x=y \\
\varepsilon \quad \text { if } x \neq y \text { and } x, y \in X \backslash A_{c} \\
\varepsilon+d(f(x), f(y)) \text { if } x \neq y \text { and } x, y \in A_{c} \\
\varepsilon+f(x) \text { if } x \in A_{c} \text { and } y \in X \backslash A_{c} \\
\varepsilon+f(y) \text { if } x \in X \backslash A_{c} \text { and } y \in A_{c}
\end{array}\right.
$$

where $d$ is defined by (3.6). It is simple to see that $\rho$ is symmetric and nonnegative. To prove the ultrametric inequality it is sufficient to show that

$$
\rho(x, y) \leqslant \rho(x, z) \vee \rho(z, y)
$$

for $x \in A_{c}, y \in X \backslash A_{c}$ and $x \neq z \neq y$. Using these relations, (3.6) and (3.8) we obtain

$$
\rho(x, y)=\varepsilon+f(x), \quad \rho(x, z) \geqslant \varepsilon+f(x) \quad \text { and } \quad \rho(z, y) \geqslant \varepsilon,
$$

so (3.9) follows. Hence, $\rho$ is an ultrametric.

Note that (3.8) and (3.7) imply (3.3). Lemma 3.7 is completely proved now.

Using the idea of spectrum of ultrametric spaces we can give the following "dual form" of Theorem 1.1. 
Theorem 3.11. Let $X$ be a finite set of points, card $X \geqslant 2$, $n$ be an integer number, $1 \leqslant n \leqslant \operatorname{card} X-1$, and let $\left\{G_{1}, \ldots, G_{n}\right\}$ be a set of complete multipartite graphs with $V\left(G_{1}\right)=\ldots=V\left(G_{n}\right)=X$ satisfying the following conditions:

$\left(i_{1}\right) G_{1}$ is complete and $G_{n}$ is nonempty in the sense that $\{x, y\} \in E\left(G_{1}\right)$ for all distinct $x, y \in X$ and $E\left(G_{n}\right) \neq \varnothing$;

(i $\left.i_{2}\right) E\left(G_{i+1}\right)$ is a proper subset of $E\left(G_{i}\right)$ for $i=1, \ldots, n-1$.

Then there is an ultrametric space $(X, \rho)$ with

$$
\operatorname{Spec}(X, \rho)=\left\{a_{0}, a_{1}, \ldots, a_{n}\right\}, 0=a_{0}<a_{1}<\ldots<a_{n}
$$

such that for $x, y \in X$

$$
\left(\{x, y\} \in E\left(G_{i}\right)\right) \Leftrightarrow\left(\rho(x, y) \geqslant a_{i}\right), \quad i=1, \ldots, n .
$$

Conversely, if $(X, \rho)$ is an ultrametric space with spectrum (3.10) and if $\left\{G_{1}, \ldots, G_{n}\right\}$ is a set of graphs such that $V\left(G_{1}\right)=\ldots=V\left(G_{n}\right)=X$ and (3.11) holds, then all $G_{i}$ are complete multipartite graphs meeting conditions $\left(i_{1}\right)$ and $\left(i_{2}\right)$.

This theorem lies beyond our considerations of diameters in ultrametric spaces so we omit the proof here.

\section{Finite ultrametric spaces with minimal sets of diametrical pairs of points}

Let $(X, d)$ be a nonempty finite ultrametric space. Let us denote by $\operatorname{DP}(X)$ the set of all ordered pairs $(x, y), x, y \in X$, for which $d(x, y)=\operatorname{diam} X$, i.e.

$$
\operatorname{DP}(X)=\{(x, y) \in X \times X: d(x, y)=\operatorname{diam} X\} .
$$

It is almost evident that the set $\operatorname{DP}(X)$ cannot be a "small" subset of $X \times X$. In this section we describe finite ultrametric spaces $(X, d)$ for which card $\operatorname{DP}(X)$ is smallest among all ultrametric spaces having the same number of elements.

We start from the following simple example. 
Example 4.1. Let $(X, d)$ be a nonempty ultrametric space, let $a$ be a point such that $a \notin X$ and let $t$ be a real number for which diam $X<t$. Write $X_{a}:=X \cup\{a\}$ and define

$$
d_{a}(x, y):=\left\{\begin{array}{l}
d(x, y) \quad \text { if } x, y \in X \\
0 \text { if } x=y=a \\
t \quad \text { if } x \in X, y=a \text { or } y \in X, x=a .
\end{array}\right.
$$

It is easy to see that $\left(X_{a}, d_{a}\right)$ is an ultrametric space and

$$
\left(d_{a}(x, y)=\operatorname{diam} X_{a}\right) \Leftrightarrow((x=a \text { and } y \in X) \text { or }(y=a \text { and } x \in X))
$$

and $\operatorname{card} D P\left(X_{a}\right)=2 \operatorname{card} X$.

Theorem 4.2. Let $(X, \rho)$ be a finite ultrametric space with card $X \geqslant 2$. Then the inequality

$$
\operatorname{card} \operatorname{DP}(X) \geqslant 2(\operatorname{card} X-1)
$$

holds. Equality in 4.1) is attained if and only if there is an ultrametric space $(Y, d)$ such that $(X, \rho)$ is isometric to $\left(Y_{a}, d_{a}\right)$.

We divided the proof of Theorem 4.2 into next two lemmas.

Lemma 4.3. Let $(X, \rho)$ be a finite ultrametric space with card $X \geqslant 2$. Then inequality (4.1) holds.

Proof. Let $x_{1}$ and $x_{2}$ be fixed points of $X$ such that $\rho\left(x_{1}, x_{2}\right)=\operatorname{diam} X$. The ultrametric inequality implies that $\rho\left(x_{1}, y\right)=\operatorname{diam} X$ or $\rho\left(x_{2}, y\right)=$ $\operatorname{diam} X$ for every $y \in X \backslash\left\{x_{1}, x_{2}\right\}$. Consequently, the number of diametrical pairs of points is greater or equal than $1+\operatorname{card}\left(X \backslash\left\{x_{1}, x_{2}\right\}\right)=\operatorname{card} X-1$. Inequality (4.1) follows.

Lemma 4.4. Let $(X, \rho)$ be a finite ultrametric space with card $X \geqslant 2$. If

$$
\operatorname{card} \mathrm{DP}(X)=2(\operatorname{card} X-1),
$$

then there is $x_{0} \in X$ such that

$$
\operatorname{diam} X=\rho\left(x_{0}, x\right)
$$

for every $x \in X \backslash\left\{x_{0}\right\}$ and $\operatorname{diam} X>\rho(x, y)$ for all $x, y \in X \backslash\left\{x_{0}\right\}$. 
Proof. Suppose that (4.2) holds. By Theorem 3.4 the set $X$ together with the set of $\rho$-diametrical pairs of points form a complete $k$-partite graph $G$ with $k \geqslant 2$. We must show that $G$ is bipartite $(k=2)$ with a bipartition $\left(X_{1}, X_{2}\right)$,

$$
X_{1} \cup X_{2}=X, \quad X_{1} \cap X_{2}=\varnothing
$$

such that card $X_{1}=1$ or $\operatorname{card} X_{2}=1$. Let $X_{1}, \ldots, X_{k}$ be a partition of $X=V(G)$. Write

$$
m_{i}=\operatorname{card} X_{i}, \quad i=1, \ldots, k
$$

and $n=\operatorname{card} X$. If $k \geqslant 3$, then using Lemma 4.3 and the equality $\sum_{i=2}^{k} m_{i}=$ $n-m_{1}$ we obtain

$$
\begin{gathered}
\operatorname{card} D P(X)=\sum_{i=1}^{k} m_{i}\left(n-m_{i}\right)>m_{1}\left(n-m_{1}\right)+\sum_{i=2}^{k} m_{i}\left(n-\sum_{i=2}^{k} m_{i}\right) \\
=m_{1}\left(n-m_{1}\right)+\left(n-m_{1}\right)\left(n-\left(n-m_{1}\right)\right) \geqslant 2(n-1) .
\end{gathered}
$$

Hence, (4.2) does not hold if $k \geqslant 3$. Thus, $k=2$, i.e. $G$ is a complete bipartite graph. Let us consider bipartition (4.4). Then $n=m_{1}+m_{2}$ and

$$
\operatorname{card} \mathrm{DP}(X)=m_{1}\left(n-m_{1}\right)+m_{2}\left(n-m_{2}\right)=2 m_{1}\left(n-m_{1}\right) .
$$

Using (4.2) we obtain

$$
2\left(n-m_{1}\right) m_{1}=2(n-1) .
$$

Quadratic equation (4.5) has the roots 1 and $n-1$. Thus, card $X_{1}=1$ or card $X_{2}=1$ as required.

Theorem 4.2 can be reformulated in the graph theory language.

Corollary 4.5. Let $G=(V, E)$ be a finite complete $k$-partite graph with $k \geqslant 2$. Then

$$
\text { card } E \geqslant \operatorname{card} V-1 \text {. }
$$

Equality in (4.6) is attained if and only if $G$ is a star.

Acknowledgment. The second author is thankful to the Finnish Academy of Science and Letters for the support. 


\section{References}

[1] V. Lemin, Finite ultrametric spaces and computer science, in: Categorical Perspectives, ed. Jurgen Koslowski, Austin Melton (Trends in Mathematics, 16), Birkhauser-Verlag, Boston, Basel, Berlin (2001), 219-242.

[2] Gunnar Carlsson, Facundo Mémoli, Characterization, Stability and Convergence of Hierarchical Clustering Methods // The Journal of Machine Learning Research, 11, 3/1 (2010), 1425-1470.

[3] V. Gurvich, M. Vyalai, Characterization of (quasi-) ultrametric finite spaces in terms of (directed) graphs // Ractor Research Report, N7, (2011), 1-23.

[4] A. J. Lemin, The category of ultrametric spaces is isometric to the category of complete, atomic, tree-like and real graduated lattices LAT* // Algebra Universalis 50 (2003), N1, 35-49.

[5] Chuang Shao, Urysohn ultrametric spaces and isometry groups, Dissertation Prepared for the Degree of Doctor of Philosophy, University of North Texas, 2009.

[6] O. Dovgoshey, O. Martio, Products of Metric Spaces, Covering Numbers, Packing Numbers and Characterizations of Ultrametric Spaces // Rev. Roumaine Math. Pures Appl., 54 (2009), N 5-6, 423-439.

[7] I. Zarichnyi, Gromov-Hausdorff Ultrametric// arXiv:math/0511437.

[8] Derong Qiu, Geometry of Non-Archimedean Gromov-Hausdorf distance// P-Adic Numbers, Ultrametric Analysis and Applications, 1 (2009), N4, 317-337.

[9] O. Dovgoshey, D. Dordovskyi, Ultrametricity and Metric Betweenness in Tangent Spaces to Metric Spaces// P-Adic Numbers, Ultrametric Analysis and Applications, 2 (2010), N2, 100-113.

[10] P. I. Balk, On an extension of the concept of metric (Russian)// Dokl. Akad. Nauk, 426 (2009), N5, 583-585; translation in Dokl. Math., 79 (2009), N3, 394-396.

[11] R. Engelking, General Topology. Revised and completed ed. (Sigma series in pure mathematics; 6), Berlin: Heldermann, 1989. 
[12] J. A. Bondy, U.S.R. Murty, Graph Theory. (Graduate Texts in Mathematics; 244), Springer, Berlin, 2008.

[13] R. Diestel, Graph Theory. Third edition. (Graduate Texts in Mathematics; 173), Springer, Berlin, 2005.

[14] C. Delhommé, C. Laflamme, M. Pouzet, N. Sauer, Indivisible ultrametric spaces // Topology Appl. 155 (2008), N14, 1462-1478.

\section{Dordovskyi}

Institute of Applied Mathematics and Mechanics of NASU, R. Luxemburg str. 74, Donetsk 83114, Ukraine

E-mail: dordovskydmitry@gmail.com

O. Dovgoshey

Institute of Applied Mathematics and Mechanics of NASU, R. Luxemburg str. 74, Donetsk 83114, Ukraine

E-mail: aleksdov@mail.ru

\section{E. Petrov}

Institute of Applied Mathematics and Mechanics of NASU, R. Luxemburg str. 74, Donetsk 83114, Ukraine

E-mail: eugeniy.petrov@gmail.com 